\title{
THE RADIAL VELOCITIES OF GALAXIES NEAR NGC 7331
}

\author{
C. R. LYNDS
}

Kitt Peak National Observatory, Tucson, Ariz., U.S.A.

\begin{abstract}
Radial velocity determinations for galaxies in the vicinity of NGC 7331, including the members of 'Stephan's Quintet', show clearly a superposition of at least two distinct physical systems. One includes NGC 7331, NGC 7320 (a Quintet member), and NGC 7343, while the other - seven or eight times more distant - contains the four remaining 'Quintet' constituents and six of the seven optical 'companions' of NGC 7331.
\end{abstract}

I report here the results of a partially completed program of radial velocity determinations for galaxies in the vicinity of NGC 7331 - a region that includes the group known as Stephan's Quintet. The motivation for the investigation and the context of this discussion have to do with the reality of the physical association of galaxies, where suggestions of such associations are based on apparent geometrical grouping. The discussion is specifically directed at two apparent groups: (a) Stephan's Quintet; and (b) NGC 7331 together with six or seven apparent companions.

Stephan's Quintet is located approximately 0.5 south-preceding NGC 7331 and consists of the galaxies NGC 7317, 7318a, 7318b, and 7319 having radial velocities in the neighborhood of $6500 \mathrm{~km} \mathrm{~s}^{-1}$ (de Vaucouleurs and de Vaucouleurs, 1964) and NGC 7320 having a velocity of approximately $800 \mathrm{~km} \mathrm{~s}^{-1}$. The highly discordant velocity of NGC 7320 was discovered by E. M. and G. R. Burbidge (1961) who also discussed the possibility of the galaxy's membership in the group and the consequent very large internal energy of the system. There appear to be two alternatives: (a) NGC 7320 is a physical member and the system is highly unstable; or (b) NGC 7320 is a foreground galaxy.

NGC 7331 is an Sb spiral galaxy of moderately large angular extent (approximately $10^{\prime}$ along the major axis) having no companions of comparable apparent size in its vicinity; the nearest comparable galaxies are more than $6^{\circ}$ distant. On the other hand, there are several galaxies of substantially smaller angular dimensions nearby, and the nearer of these together with NGC 7331 constitutes one of the 174 systems discussed by Holmberg (1969) as possible physical systems. Holmberg notes seven companions to NGC 7331 within a radius of approximately $16^{\prime}$ and concludes that, on a statistical basis, four among these are likely to be physically associated with the larger galaxy. However, one of the apparent companions, NGC 7335, is known to have a radial velocity near $6300 \mathrm{~km} \mathrm{~s}^{-1}$, as compared with the much smaller value of approximately $800 \mathrm{~km} \mathrm{~s}^{-1}$ for NGC 7331 (de Vaucouleurs and de Vaucouleurs, 1964). The only other galaxy in the field to have a published radial velocity is NGC 7343; it has a velocity of about $1200 \mathrm{~km} \mathrm{~s}^{-1}$ but is outside Holmberg's survey area.

The similarity between the radial velocities of NGC 7331, NGC 7343, and the discordant member of Stephan's Quintet, on the one hand, and between the velocities of the other members of the Quintet and that of one of the optical companions of 
NGC 7331, on the other, led me to investigate further the possibility that the distribution of galaxies in the field results from the superposition of a nearby and a distant group of galaxies. To this end, radial velocities have been determined for several of the numerous other galaxies in the field. These observations, together with the few existing determinations already mentioned, are indicated in Figure 1. This illustration shows a $1.7 \times 1.7$ field centered near the large spiral galaxy NGC 7331 ; the radial velocities are given to two significant figures and are uncorrected for the rotation of our galaxy.

A careful inspection of the field reveals what appears to be a large association of

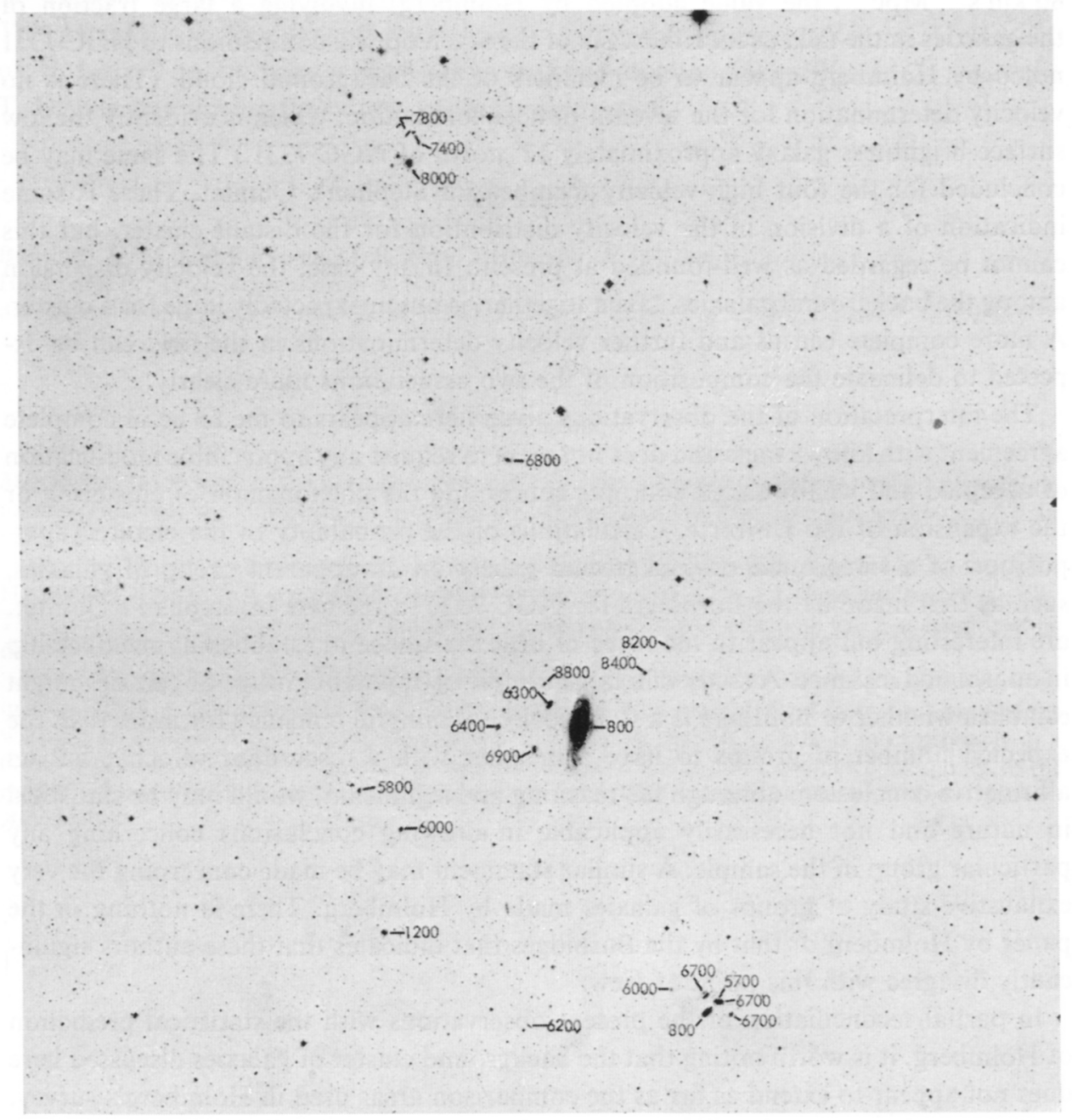

Fig. 1. A $1^{\circ} .7 \times 1^{\circ} .7$ field centered near the large spiral galaxy NGC 7331 . North is at the top; East is toward the left. The radial velocities that are known for galaxies in the field are indicated to two significant figures. 
galaxies having apparent dimensions of the order of those of the members of Stephan's Quintet and the optical companions of NGC 7331. It will also be noted that the radial velocities for those galaxies that have been observed show a marked segregation into two groups: one at about $1000 \mathrm{~km} \mathrm{~s}^{-1}$ which includes NGC 7331, NGC 7343, and NGC 7320; and one at about $7000 \mathrm{~km} \mathrm{~s}^{-1}$ which includes the remaining 18 galaxies.

The simplest and most straightforward interpretation of the observations is that there are two distinct physical groups of galaxies: (a) NGC 7331, together with two confirmed physical companions, NGC 7320 and NGC 7343, at a distance of $11 \mathrm{Mpc}$ (based on the distance modulus of 30.2 adopted by Holmberg for NGC 7331); and (b) a background cloud at approximately $90 \mathrm{Mpc}$ (based on a Hubble parameter of $80 \mathrm{~km} \mathrm{~s}^{-1} \mathrm{Mpc}^{-1}$, the value adopted by Holmberg) involving a large fraction of the galaxies in the field as members. Six of the seven optical companions to NGC 7331 noted by Holmberg appear to be members of the background cloud. (There is no velocity determination for the seventh optical companion, which is evidently the low surface-brightness galaxy approximately $12^{\prime}$ north of NGC 7331.) The same may be concluded for the four high-velocity members of Stephan's Quintet. There is some indication of a division in the velocity distribution for the distant cluster, but this cannot be regarded as well-founded at present. In any case, the velocity dispersion among the background galaxies, taken together, is not unexpectedly large for a cluster. A more complete census and further velocity determinations in the field can be expected to delineate the composition of the two associations more clearly.

The interpretation of the observations given here appears to me to be in complete agreement with known facts and does not seem to require any appreciable modification of accepted and well-founded concepts concerning the phenomenon of clustering or the expansion of the Universe. Calculations of the probability of the chance superposition of a foreground or background galaxy on an apparent group of galaxies, such as that made by the Burbidges for NGC 7320 in the case of Stephan's Quintet, are interesting but appear to me to be of little assistance in establishing membership in an isolated instance. A study of a large sample of apparent groups of galaxies might establish whether or not there is a statistically meaningful tendency for more than the expected number of groups to have a member with a discordant velocity, but an affirmative conclusion, although far-reaching and significant, would only be statistical in nature and not necessarily applicable in drawing conclusions concerning any particular group in the sample. A similar statement may be made concerning the very exhaustive study of groups of galaxies made by Holmberg. There is nothing in the paper by Holmberg or that by the Burbidges that indicates that these authors significantly disagree with this point of view.

In partial reconciliation of the present observations with the statistical prediction of Holmberg, it is worth noting that the background cluster of galaxies discussed here does not appear to extend as far as the comparison areas used in Holmberg's survey. And, in connection with what I now prefer to call 'Stephan's Quartet', a certain amount of 'sub-clustering' may well be a characteristic of the background cluster, for it will be noticed that the three northernmost galaxies for which velocities were 
determined appear to be members of a compact apparent septet that may later be established as a physical group. However, even if all members of this group have similar radial velocities, there is no assurance that some of the apparent members are not simply members of the larger cloud projected on the group.

The essence of the method applied in this investigation has been simply the addition, through the Hubble law, of radial distance to the two tangential coordinates as a metric dimension in which the observed distribution of galaxies may be used as a clustering criterion.

\section{Acknowledgements}

This paper was written while the author was visiting the Astronomy Centre, University of Sussex. The author acknowledges helpful discussions with H. C. Arp and T. D. Kinman during the course of this investigation.

\section{References}

Burbidge, E. M. and Burbidge, G. R.: 1961, Astrophys. J. 134, 244.

Holmberg, E.: 1969, Arkiv Astron. 5, No. 20.

de Vaucouleurs, G. de Vaucouleurs, A.: 1964, Reference Catalog of Bright Galaxies, University of Texas Press, Austin.

\section{Discussion}

Mrs Rubin: Is the scale of the galaxies in Stephan's Quintet sufficient to study the stellar population, or other distance discriminants, to establish the distances of the galaxies, independently of their velocities?

Mrs Burbidge: It occurred to Geoff and me that it should be possible to look at the diameters of the Hur regions in NGC 7331 and NGC 7320; NGC 7331 is of similar redshift to the Virgo cluster galaxies. There might be other discriminants that could be used in the classifications by van den Bergh and by de Vaucouleurs.

Allen: Using the large radio telescope at Nançay, we have succeeded in observing neutral hydrogen in NGC 7320. I have compared the hydrogen mass and total mass derived from the observations with the values obtained on other Sbc galaxies. The result is that NGC 7320 is most probably at a distance of about $10 \mathrm{Mpc}$, corresponding to its redshift, and not at $67 \mathrm{Mpc}$ which corresponds to the redshift of the other four members of Stephan's Quintet. (This research will be published in Astron. Astrophys.). 